

\title{
Factorization versus Duality in Nonleptonic decays, a Quark Model Approach.
}

\author{
A. Le Yaouanc, L. Oliver, O. Pène and J.-C. Raynal
}

August 9, 2018

Laboratoire de Physique Théorique et Hautes Energies"

Université de Paris XI, Bâtiment 211, 91405 Orsay Cedex, France

\section{Abstract}

\begin{abstract}
We study in a quark model the contradiction between factorization and duality found in nonleptonic decays at next to leading order in $1 / N_{c}$, concentrating on quark exchange mechanism. The contradiction originates in the fact that the standard factorization assumption approximates the asymptotic final states by a non-orthogonal set of states, thus leading to an overcounting of the decay probability. We consider a system with two heavy quarks treated as classical color sources with constant velocity, and two mass-degenerate antiquarks. Exploiting permutation symmetry in an adiabatic approximation, we find that final state interaction restores duality. Three $O\left(1 / N_{c}\right)$ effects are exhibited: i) a proper treatment of orthogonality yields a global correction $1 / N_{c} \rightarrow 1 / 2 N_{c}$ within a generalized factorization à la BSW, (such a factor was present in an Ansatz by Shifman), ii) the distorsion of the meson wave functions at the time of the weak decay, iii) relative phases generated by the later evolution. The latter effect becomes dominant for light antiquarks or for a small velocity of the final mesons, and may thoroughly modify the factorization picture. For exclusive decay it may interchange the role of class I and class II final channels, and for semi-inclusive decay it may lead to an equal sharing of the probability between the two sets of final states. In the heavy antiquark and large velocity limit, the replacement $1 / N_{c} \rightarrow 1 / 2 N_{c}$ is the dominant correction.
\end{abstract}

LPTHE-Orsay 95/26,

hep-ph/9504262

\footnotetext{
${ }^{1}$ Laboratoire associé au Centre National de la Recherche Scientifique - URA D00063
} 


\section{Introduction.}

While some progress has been performed during these last years in our understanding of semileptonic and leptonic decay mechanisms, our understanding of nonleptonic decays is still semi-quantitative. Not to speak of the $\Delta I=1 / 2$ mystery, the nonleptonic decays of $D$ and $B$ mesons are most often studied with the help of the standard factorization assumption [1], the theoretical basis for which exists only in the $N_{c} \rightarrow \infty$ limit, or of the generalized factorization assumption à la BSW [2] which is a phenomenological Ansatz.

The nonleptonic decay channels of heavy meson are an important issue and will grow even more so, since they provide the cannels in which the CP asymmetries will be looked for in $B$-factories.

A critical study of factorization assumption is an urgent task, and has indeed been started [3], at a time when increasingly accurate experimental results learn us that our present understanding of $B \rightarrow \psi K\left(K^{*}\right)$, based on factorization assumption among other hypotheses, severely fails $\llbracket 4$.

It is usually claimed that the corrections to factorization are due to final state interaction (FSI). In a sense this statement is true, but the proper meaning of what is understood by that needs clarification. One of our aims in this paper is to proceed to this clarification in a simple model in which the dynamics is rather transparent.

Furthermore little is known about the validity of factorization except that it is violated in low energy $K \rightarrow \pi \pi$ and $D \rightarrow K \pi, K K, \pi \pi$ [5] [6] channels where strong FSI phases are experimentally known to be present, both from the direct analysis

of weak nonleptonic decays and from scattering experiments. The study grows more difficult at larger energies when more channels are coupled. This happens when multiparticle channels come in, particularly multi-pion channels, and also when several two-body channels communicate via strong interaction through quark exchange or quark-pair annihilation-creation.

We will concentrate on two-body channels communicating via quark exchange. In a Tamm-Dancoff type expansion, quark exchange is dominant since it needs no quark pair creation neither annihilation. Furthermore, it has been stressed by J. Donoghue [7] that such a mechanism might explain the $D^{0} \rightarrow \phi \bar{K}^{0}$ decay amplitude.

On the other hand, M. Shifman [8] (see also [9]) has made the interesting remark that beyond leading $1 / N_{c}$ order, the factorization assumption simply violates duality. As we shall show at length in this paper, this effect is fully related to final state interaction via quark exchange.

Indeed this comes from the fact that in color space a $q q \bar{q} \bar{q}$ color singlet can be decomposed in two ways into two $q \bar{q}$ color singlets, but the two resulting states are not orthogonal to each other [9]. At the time the weak decay of the initial meson creates a $q q \bar{q} \bar{q}$ color singlet, the four quarks interact strongly with one another. It is impossible to tell that one pair $q \bar{q}$ is in one meson and the other pair in another meson. However the system evolves, and eventually splits into two $q \bar{q}$ color singlets that are 
spatially distant and hence orthogonal. During all the period of the interaction, one given quark does not know with which antiquark it is paired. This is exactly the situation which is expressed usually by the expression "quark exchange". What is depicted by this expression is not a simple and instantaneous exchange of a quark from one meson to another, but a period of overlap of the two mesons resulting finally in a non-vanishing amplitude for an exchange of a quark.

The factorization assumption totally overlooks this complex mechanism, as it simply computes the overlap of the $q q \bar{q} \bar{q}$ system resulting from weak decay with the final mesons. Two non-orthogonal states are taken as an approximation of two distinct final states which are obviously orthogonal. Once one realizes this, it is not a surprise that one encounters some problems with probability conservation.

Our aim is mainly to understand better this interaction mechanism in a simple model, to check that when the dynamics is correctly treated there is no contradiction with duality, and to identify the different effects contributing at next to leading order in $1 / N_{c}$.

We will work in the kinematical situation considered by Shifman [8], in a Hamiltonian approach, namely a quark model one. In section 2. we will rephrase duality in the language of closure theorem and reexpress the contradiction between factorization and duality. In section 3 we will present a quark model, with an adiabatic approximation and mass-degenerate antiquarks, and we exploit the resulting permutation symmetry to compute the $\mathrm{S}$ matrix and the weak decay amplitude. In section 4 we will conclude.

\section{Duality versus factorization, rephrasing the prob- lem.}

Let us recall the ideal process which is studied in [8]. There are three heavy quarks $A, B, C$ and two light antiquarks $\bar{\alpha}, \bar{\beta}$. We will assume the latter to be heavy enough to justify the use of the quark potential model which will be our tool all along.

The process under study is the weak decay

$$
P_{A \bar{\alpha}} \rightarrow M_{B \bar{\alpha}}+M_{C \bar{\beta}}, M_{B \bar{\beta}}+M_{C \bar{\alpha}}
$$

where $P_{A \bar{\alpha}}$ is pseudoscalar meson composed by $A$ and $\bar{\alpha}$ and $M_{B \bar{\alpha}}$ represents any meson composed by $B$ and $\bar{\alpha}$.

The following relations are assumed [8]:

$$
M_{B}=M_{C} \equiv M, \quad M_{A}=2 M+\Delta, \quad \Lambda_{Q C D} \ll \Delta \ll M
$$

To which we add

$$
\Lambda_{Q C D} \ll m_{\alpha}, m_{\beta}
$$

to justify the use of quark model. 
We cannot find a physical example of such a situation. If the $s$ quark was heavy, the ideal situation assumed in [8] would be realized by the couple of decay channels: $B_{d} \rightarrow D^{0} \bar{K}^{0}, D^{+} K^{-}$.

\subsection{Relation between duality and closure theorem.}

In [8] M. Shifman exhibits a contradiction between duality and the standard factorization hypothesis also encountered in [9] while studying $\Delta \Gamma$ for the $B_{s}-\bar{B}_{s}$ system. We will study this issue as a contradiction between the closure theorem and the standard factorization hypothesis. Indeed, duality is related to the closure theorem in quantum mechanics as we will now recall.

Let us call generically $\mid n>$ all hadronic states built up with quarks $B, C, \bar{\alpha}, \bar{\beta}$. Calling $H_{W}$ the weak Hamiltonian, the state $H_{W} \mid P_{A \bar{\alpha}}>$ is composed of the four quarks $B, C, \bar{\alpha}, \bar{\beta}$. The decay width of the $P_{A \bar{\alpha}}$ meson is given by

$$
\begin{aligned}
\Gamma\left(P_{A \bar{\alpha}}\right) & =\sum_{n}<P_{A \bar{\alpha}}\left|H_{W}\right| n><n\left|H_{W}\right| P_{A \bar{\alpha}}>\delta\left(E_{n}-E_{A \bar{\alpha}}\right) \\
& =\sum_{n}<P_{A \bar{\alpha}}\left|H_{W}\right| n>\delta\left(H-E_{A \bar{\alpha}}\right)<n\left|H_{W}\right| P_{A \bar{\alpha}}>
\end{aligned}
$$

where $E_{A \bar{\alpha}}$ is the initial energy, $E_{n}$ is the energy of the state $|n\rangle, H$ is the strong Hamiltonian $\left(H\left|n>=E_{n}\right| n>\right)$, and where the sum is to be understood as a sum over discrete states and an integral over continuum states. The set of states $|n\rangle$ is a complete set. The sum could be expanded on any basis, and in particular on the basis of the free quarks $B, C, \bar{\alpha}, \bar{\beta}$. This is where the closure of the Hilbert space comes in.

Now, $H=H_{c}+V$ where $H_{c}$ stands for the kinetic energy and $V$ for the potential. Whenever the contribution from $V$ can be neglected in front of $H_{c}$, one recovers lowest order duality, i.e. the simple parton model with no perturbative corrections neither non-perturbative ones from higher dimension operators. We do not want to go into the question of when this approximation is valid, and how to get better approximations. We simply want to rephrase the contradiction between factorization and duality as a contradiction between factorization and closure, then go into a simple model to show how the dynamics solves this.

\subsection{Contradiction between factorization and closure.}

Let us consider for example the weak Hamiltonian:

$$
H_{W}=2 \sqrt{2} G\left[C_{1}\left(\bar{B} \gamma_{\mu} L A\right)\left(\bar{C} \gamma^{\mu} L \beta\right)+C_{2}\left(\bar{B} \gamma_{\mu} L \beta\right)\left(\bar{C} \gamma^{\mu} L A\right)\right]
$$

where $L=\left(1-\gamma_{5}\right) / 2, C_{1}$ and $C_{2}$ are coefficients that we do not need to specify in this paper, although they are reminiscent of the familiar coefficients in the effective weak interaction Hamiltonian. 
At the time $t=0$ the weak Hamiltonian acts on the initial meson and produces a state that contains the quarks $C, \bar{\beta}, \bar{\alpha}, B$. Let us call this state $\mid f>$. We find it convenient for later use to decompose $\mid f>$ into its color part and the remainder:

$$
\begin{aligned}
& \left|f>\equiv H_{W}\right| P_{A \bar{\alpha}}>=\sum_{s_{C}, s_{\bar{\beta}}, c_{C}, c_{\bar{\beta}},} \int d \vec{p}_{C} d \vec{p}_{\bar{\beta}} d \vec{p}_{B} d \vec{p}_{\bar{\alpha}} \Psi\left(\vec{p}_{C}, s_{C}, \vec{p}_{\bar{\beta}}, s_{\bar{\beta}}, \vec{p}_{B}, s_{B}, \vec{p}_{\bar{\alpha}}, s_{\bar{\alpha}}\right) \\
& s_{B}, s_{\bar{\alpha}}, c_{B}, c_{\bar{\alpha}} \\
& \frac{1}{N_{c}}\left[C_{1} \delta_{c_{C}, c_{\bar{\beta}}} \delta_{c_{B}, c_{\bar{\alpha}}}+C_{2} \delta_{c_{C}, c_{\bar{\alpha}}} \delta_{c_{B}, c_{\bar{\beta}}}\right] \mid C, \vec{p}_{C}, s_{C}, c_{C} ; \bar{\beta}, \vec{p}_{\bar{\beta}}, s_{\bar{\beta}}, c_{\bar{\beta}} ; B, \vec{p}_{B}, s_{B}, c_{B} ; \bar{\alpha}, \vec{p}_{\bar{\alpha}}, s_{\bar{\alpha}}, c_{\bar{\alpha}}>(5)
\end{aligned}
$$

where $\vec{p}_{C}, s_{C}, c_{C}\left(\vec{p}_{\bar{\beta}}, s_{\bar{\beta}}, c_{\bar{\beta}}\right)$ labels the momentum, spin and color of the (anti-)quark $C(\bar{\beta}), N_{c}$ is the number of colors. The function $\Psi$ may be computed from the wave function of $P_{A \bar{\alpha}}$ and the operator $H_{W}$ in (4). However we will skip this computation since the precise expression for $\Psi$ is not relevant for our argument.

It is obvious that:

$$
<f \mid f>=\left(C_{1}^{2}+C_{2}^{2}+2 \frac{C_{1} C_{2}}{N_{c}}\right) K
$$

with

$$
K=\sum_{\substack{s_{C}, s_{\bar{\beta}}, c_{C}, c_{\bar{\beta}}, s_{B}, s_{\bar{\alpha}}, c_{B}, c_{\bar{\alpha}}}} \int d \vec{p}_{C} d \vec{p}_{\bar{\beta}} d \vec{p}_{B} d \vec{p}_{\bar{\alpha}}\left|\Psi\left(\vec{p}_{C}, s_{C}, \vec{p}_{\bar{\beta}}, s_{\bar{\beta}}, \vec{p}_{B}, s_{B}, \vec{p}_{\bar{\alpha}}, s_{\bar{\alpha}}\right)\right|^{2}
$$

Let us also decompose the meson wave function into a color part and the remainder:

$$
\left|M_{C \bar{\beta}}^{(n)}>=\sum_{s_{C}, s_{\bar{\beta}}, c_{C}, c_{\bar{\beta}}} \int d \vec{p}_{C} d \vec{p}_{\bar{\beta}} \psi_{C \bar{\beta}}^{(n)}\left(\vec{p}_{C}, s_{C}, \vec{p}_{\bar{\beta}}, s_{\bar{\beta}}\right) \frac{1}{N_{C}^{\frac{1}{2}}} \delta_{c_{C}, c_{\bar{\beta}}}\right| C, \vec{p}_{C}, s_{C}, c_{C} ; \bar{\beta}, \vec{p}_{\bar{\beta}}, s_{\bar{\beta}}, c_{\bar{\beta}}>
$$

and analogously for all quark-antiquark pairs. The $\psi_{C \bar{\beta}}^{(n)}$ 's form a complete orthonormal basis of the spin-momentum $C \bar{\beta}$ Hilbert space. Let us now define the spin-space overlaps:

$$
\begin{gathered}
K_{C \bar{\beta} ; B \bar{\alpha}}^{(n, m)}=\sum_{s_{C}, s_{\bar{\beta}}, s, s_{\bar{\alpha}}} \int d \vec{p}_{C} d \vec{p}_{\bar{\beta}} d \vec{p}_{B} d \vec{p}_{\bar{\alpha}} \Psi^{\dagger}\left(\vec{p}_{C}, s_{C}, \vec{p}_{\bar{\beta}}, s_{\bar{\beta}}, \vec{p}_{B}, s_{B}, \vec{p}_{\bar{\alpha}}, s_{\bar{\alpha}}\right) \\
\psi_{C \bar{\beta}}^{(n)}\left(\vec{p}_{C}, s_{C}, \vec{p}_{\bar{\beta}}, s_{\bar{\beta}}\right) \psi_{B \bar{\alpha}}^{(m)}\left(\vec{p}_{B}, s_{B}, \vec{p}_{\bar{\alpha}}, s_{\bar{\alpha}}\right)
\end{gathered}
$$

and analogously for the alternative grouping of quark-antiquark pairs: $C \bar{\alpha} ; B \bar{\beta}$. Closure in the $C \bar{\beta}$ and $B \bar{\alpha}$ spin-momentum subspaces implies that

$$
\sum_{n, m} K_{C \bar{\beta} ; B \bar{\alpha}}^{(n, m) *} K_{C \bar{\beta} ; B \bar{\alpha}}^{(n, m)}=K
$$


and analogously

$$
\sum_{n, m} K_{B \bar{\beta} ; C \bar{\alpha}}^{(n, m) *} K_{B \bar{\beta} ; C \bar{\alpha}}^{(n, m)}=K
$$

From eqs. (5), (8) and (9) it results that

$$
\begin{array}{ll}
<f \mid M_{C \bar{\beta}}^{(n)} ; M_{B \bar{\alpha}}^{(m)}>= & a_{1} K_{B \bar{\beta} ; C \bar{\alpha}}^{(n, m)} \\
<f \mid M_{B \bar{\beta}}^{(n)} ; M_{C \bar{\alpha}}^{(m)}>= & a_{2} K_{B \bar{\beta} ; C \bar{\alpha}}^{(n, m)} .
\end{array}
$$

with

$$
a_{1}=C_{1}+\frac{C_{2}}{N_{c}}, \quad a_{2}=C_{2}+\frac{C_{1}}{N_{c}}
$$

Up to now all equations were exact. Now we shall formulate in our formalism the factorization approximation by assuming that the decay amplitudes are well approximated by the overlaps:

$$
\begin{aligned}
T\left(P_{A \bar{\alpha}} \rightarrow M_{C \bar{\beta}}^{(n)} M_{B \bar{\alpha}}^{(m)}\right) & \simeq<f \mid M_{C \bar{\beta}}^{(n)} M_{B \bar{\alpha}}^{(m)}>^{*} \\
T\left(P_{A \bar{\alpha}} \rightarrow M_{B \bar{\beta}}^{(n)} ; M_{C \bar{\alpha}}^{(m)}\right) & \simeq<f \mid M_{B \bar{\beta}}^{(n)} M_{C \bar{\alpha}}^{(m)}>^{*}
\end{aligned}
$$

More precisely, the standard factorization assumption [1] uses eqs. (12) (13) and (14) with $C_{1}, C_{2}$ computed from the electroweak theory complemented with QCD radiative corrections. Bauer, Stech and Wirbel [2] have proposed a phenomanological factorization assumption that keeps eqs. (12) and (14) but with $a_{1}$ and $a_{2}$ fitted to all known $D$, respectively $B$, decays.

Within standard factorization, summing over all two meson final states and using eqs. (5), (7), (10), (11), (12) (13) and (14) we get:

$$
\sum_{n, m}\left|T\left(P_{A \bar{\alpha}} \rightarrow M_{C \bar{\beta}}^{(n)} M_{B \bar{\alpha}}^{(m)}\right)\right|^{2}+\left|T\left(P_{A \bar{\alpha}} \rightarrow M_{B \bar{\beta}}^{(n)} M_{C \bar{\alpha}}^{(m)}\right)\right|^{2}=\left(\left(C_{1}^{2}+C_{2}^{2}\right)\left(1+\frac{1}{N_{c}^{2}}\right)+4 \frac{C_{1} C_{2}}{N_{c}}\right) K
$$

To leading order in $1 / N_{c}$ eq. (15) gives the same result as eq. (6). In our present framework this reflects the well known fact that to leading order in $1 / N_{c}$ factorization and duality are compatible. However, the $O\left(1 / N_{c}\right)$ corrections show a discrepancy, the contradiction stressed in [8]. As suggested in [8], this discrepancy could be cured, except for the $1 / N_{c}^{2}$ terms by using a phenomenological factorization with

$$
a_{1}=C_{1}+\frac{C_{2}}{2 N_{c}}, \quad a_{2}=C_{2}+\frac{C_{1}}{2 N_{c}}
$$

However, in our framework it is easy to trace back the origin of the discrepancy. The fact is that the set of states $\left|M_{C \bar{\beta}}^{(n)} M_{B \bar{\alpha}}^{(m)}>\oplus\right| M_{B \bar{\beta}}^{(n)} M_{C \bar{\alpha}}^{(m)}>$ is not an orthonormal basis of the Hilbert space. The states are normalised, but they are not orthogonal: 


$$
<M_{C \bar{\beta}}^{(n)} M_{B \bar{\alpha}}^{(m)} \mid M_{B \bar{\beta}}^{(n)} M_{C \bar{\alpha}}^{(m)}>=O\left(\frac{1}{N_{c}}\right) \neq 0
$$

in general. This overlap is $O\left(1 / N_{c}\right)$ as can be easily derived from the color part in wave function (8):

$$
\left(\frac{1}{N_{c}^{\frac{1}{2}}}\right)^{4} \sum_{C_{C}, C_{B}, C_{\bar{\alpha}}, C_{\bar{\beta}}} \delta_{C_{C}, C_{\bar{\beta}}} \delta_{C_{B}, C_{\bar{\alpha}}} \delta_{C_{B}, C_{\bar{\beta}}} \delta_{C_{C}, C_{\bar{\alpha}}}=\frac{1}{N_{c}}
$$

It is easy to check that (17) is at the origin of the discrepancy between (15) and (6).

Neither are these states eigenstates of the strong Hamiltonian. Indeed, these states are built up from two asymptotic mesons combined via a plane wave for the relative momentum between the two mesons. When the two mesons lie far apart, the simple product of their wave function is an eigenstate of the strong Hamiltonian. However, in the states we consider there is a non negligible contribution with the two mesons overlapping in space where they strongly interact leading to an important distortion from the simple product of asymptotic meson wave functions.

\section{An Adiabatic Quark Model with Degenerate An- tiquarks.}

\subsection{The S matrix}

Let us first consider an oversimplified model. We will assume all quarks to be spinless and $\bar{\alpha}$ and $\bar{\beta}$ to be degenerate in mass: $m_{\bar{\alpha}}=m_{\bar{\beta}} \equiv m$. Next, $B$ and $C$ being very heavy, we will treat their motion as classical. They are supposed to move head-on with velocity $\vec{v}$. As a function of time $t$ the spatial coordinates of $B$ and $C$ are

$$
\vec{r}_{B}=-\vec{r}_{C}=\vec{v} t
$$

From now on the mesons will be assumed to have their center-of-mass localised in configuration space, at the position of the heavy quark and we will neglect $O(1 / M)$ corrections. The fact that the heavy quarks meet at the origin, i.e. that their impact parameter is zero means that our model will describe the $\mathrm{S}$ wave channel.

Concerning the Hamiltonian for the antiquarks $\bar{\beta}$ and $\bar{\alpha}$ we will use a color potential introduced in [10]:

$$
\begin{gathered}
H(t)=\frac{p_{\bar{\beta}}^{2}}{2 m}+\frac{p_{\bar{\alpha}}^{2}}{2 m}+\sum_{a} \lambda_{B}^{a} \lambda_{\bar{\alpha}}^{a} V\left(\vec{r}_{\bar{\alpha}}-\vec{v} t\right)+\lambda_{C}^{a} \lambda_{\bar{\alpha}}^{a} V\left(\vec{r}_{\bar{\alpha}}+\vec{v} t\right)+\lambda_{B}^{a} \lambda_{\bar{\beta}}^{a} V\left(\vec{r}_{\bar{\beta}}-\vec{v} t\right) \\
\lambda_{C}^{a} \lambda_{\bar{\beta}}^{a} V\left(\vec{r}_{\bar{\beta}}+\vec{v} t\right)+\lambda_{\bar{\alpha}}^{a} \lambda_{\bar{\beta}}^{a} V\left(\vec{r}_{\bar{\beta}}-\vec{r}_{\bar{\alpha}}\right)+\lambda_{C}^{a} \lambda_{B}^{a} V(2 \vec{v} t)
\end{gathered}
$$


where $\lambda_{C}^{a}$ is the Gell-Mann $S U(3)$ Hermitean matrix applying to quark $C$, etc, and where $-V(\vec{r})$ is a rotation-invariant confining potential, so that color singlet mesons are bound together (the $\lambda^{a} \lambda^{a}$ factor is negative on a singlet). The Hamiltonian is bounded from below when restricted to overall color-singlet states.

$H(t)$ in $(20)$ is invariant for the Permutation : $P \equiv \bar{\alpha} \leftrightarrow \bar{\beta}$. It results that all eigenstates of $H$ will be eigenstates of $P$ with eigenvalue \pm 1 . The asymptotic states, when $T \rightarrow \pm \infty$, are built from simple products of the mesonic wave functions whose center of mass are located at $\pm \vec{v} T$ :

$$
\sqrt{2}\left|D^{ \pm, n, m}, T>\underset{T \rightarrow \pm \infty}{=}\right| M_{C \bar{\beta}}^{(n)}(-\vec{v} T)>\otimes\left|M_{B \bar{\alpha}}^{(m)}(\vec{v} T)> \pm\right| M_{C \bar{\alpha}}^{(n)}(-\vec{v} T)>\otimes \mid M_{B \bar{\beta}}^{(m)}(\vec{v} T)>
$$

where the $M^{(n)}(\vec{r})$ states are the mesons states defined from eq. (\&) by a Fourier transform on the center of mass variable. We will assume the evolution in time to be adiabatic, i.e. we assume that the state $\mid D^{ \pm, n, m}, t>$ evolves in time by remaining an eigenstate of $H(t)$ for all $t$. We will further assume that during the evolution, the fundamental states $\mid D^{ \pm, 0,0}, t>$ never cross other states. It results that the twodimensional subspace spanned by $\mid D^{ \pm, 0,0}, t>$ is stable under the action of the strong Hamiltonian, i.e. that any state within this subspace evolves into a state within this subspace at a later time. Consequently, the two-by-two restriction of the S-matrix to this subsapce has to be unitary. We will now restrict ourselves to the study of the strong interaction scattering process of these two fundamental states. At time $t$, the eigenstates verify:

$$
H(t)\left|D^{ \pm, 0,0}, t>=E^{ \pm}(v t)\right| D^{ \pm, 0,0}, t>
$$

where we made use of the fact that $H$ depends on $t$ only through the product $v t$. The interaction between the two terms in the r.h.s. of (21) is $O\left(1 / N_{c}\right)$ as already argued, see (18). Hence

$$
E^{+}(v t)-E^{-}(v t)=O\left(\frac{1}{N_{c}}\right)
$$

Asymptotically it is also obvious that

$$
E^{+}( \pm \infty)=E^{-}( \pm \infty)
$$

since the two mesons do not overlap implying that the two terms in the r.h.s. of (21) become orthogonal.

In fact the two energies differ from zero only when the two mesons overlap and the overlap falls off exponentially when $v t \rightarrow \infty$. In the basis $\mid D^{ \pm, 0,0}, \pm \infty>$, (21), the $S$ matrix is diagonal and, being unitary its general form writes:

$$
S=e^{2 i \delta}\left(\begin{array}{cc}
e^{2 i \phi} & 0 \\
0 & e^{-2 i \phi}
\end{array}\right)
$$


where

$$
\begin{gathered}
2 \delta=-\int_{-\infty}^{+\infty} \frac{d z}{v}\left[\frac{E^{+}(z)+E^{-}(z)}{2}-\frac{E^{+}(\infty)+E^{-}(\infty)}{2}\right] \\
2 \phi=-\int_{-\infty}^{+\infty} \frac{d z}{v} \frac{E^{+}(z)-E^{-}(z)}{2} .
\end{gathered}
$$

Indeed, the S-matrix is given by

$$
S=T \exp \left[-i \int_{-\infty}^{\infty} d t H_{I}(t)\right]
$$

which in our case of a state, say $\mid+>$, that remains eigenstate of a time dependent Hamiltonian with energy $E^{+}(t)$, simplifies to

$$
S_{++}=\exp \left[-i \int_{-\infty}^{\infty} d t\left(E^{+}(t)-E^{+}(\infty)\right]\right.
$$

where the interaction Hamiltonian has been taken to be the total Hamiltonian minus the Energy of two non-interacting mesons.

In the meson-meson basis:

$$
\left|M_{C \bar{\beta}}^{(0)}(-\vec{v} T)>\otimes\right| M_{B \bar{\alpha}}^{(0)}(\vec{v} T)>\quad\left|M_{C \bar{\alpha}}^{(0)}(-\vec{v} T)>\otimes\right| M_{B \bar{\beta}}^{(0)}(\vec{v} T)>
$$

the $S$ matrix writes:

$$
S=e^{2 i \delta} e^{2 i \phi \sigma_{1}}=e^{2 i \delta}\left(\begin{array}{cc}
\cos 2 \phi & i \sin 2 \phi \\
i \sin 2 \phi & \cos 2 \phi
\end{array}\right)
$$

This matrix is, as expected, unitary and invariant for the permutation $P$ which permutes the lines and the columns of the matrix. When the angle $\phi$ does not vanish, there is a quark exchange between the mesons which becomes maximal for $\phi=\pi / 4$. Actually, $\phi=O\left(1 / N_{c}\right)$ from eq. (23). From eq. (26), we see that $\phi \propto 1 / v$. The reason for this is clear: the lower the velocity, the longer the mesons overlap and can exchange quarks. We will now return in our model to the contradiction discussed in the preceeding section between duality and factorization. We work out an illustrative example of these features in the Appendix.

\subsection{Final state interaction in our model.}

In this section we return to the weak interaction. We consider here the exclusive decay channels $P_{A \bar{\alpha}} \rightarrow M_{B \bar{\alpha}}^{(0)} M_{C \bar{\beta}}^{(0)}$ and $P_{A \bar{\alpha}} \rightarrow M_{C \bar{\alpha}}^{(0)} M_{B \bar{\beta}}^{(0)}$. Thanks to the statement made in the preceeding section that the subspace spanned by these two final states is stable for the weak interactions, we can safely forget all other channels in our study of the FSI.

We now assume that the weak Hamiltonian acts at $t=0$, creating the heavy quarks $B$ and $C$ at $\vec{r}=0$. The weak interaction creates a state $\mid f>$ as defined 
in (5). When $t=0$ an additional symmetry is present in the strong Hamiltonian $H(0)$ : invariance under permutation of color labels $C_{C} \leftrightarrow C_{B}$ and consequently under $C_{\bar{\beta}} \leftrightarrow C_{\bar{\alpha}}$. It results that the $\mid D^{ \pm, 0,0}, 0>$ states are even (odd) under the color permutation $P_{c}: C_{C} \leftrightarrow C_{B}$. Restricted to the color-even (color-odd) sector the Hamiltonian $H(0)$ reduces to:

$$
H(0)=\frac{p_{\bar{\beta}}^{2}}{2 m}+\frac{p_{\bar{\alpha}}^{2}}{2 m}-\frac{2\left(N_{c} \pm 2\right)\left(N_{c} \mp 1\right)}{N_{c}}\left(V\left(\vec{r}_{\bar{\alpha}}\right)+V\left(\vec{r}_{\bar{\beta}}\right)\right) \pm \frac{2\left(N_{c} \mp 1\right)}{N_{c}} V\left(\vec{r}_{\bar{\beta}}-\vec{r}_{\bar{\alpha}}\right)
$$

where the upper (lower) sign corresponds to color-even (color-odd) states.

It is important to notice that to leading order in $N_{c}$ the Hamiltonian (31) is the same for color-even and color-odd states. It results that in the $N_{c} \rightarrow \infty$ limit, the two color wave funtion multiply the same spatial wave function for $t=0$. Furthermore, to leading order in $N_{c}$ the Hamiltonian (31) is equal to twice the Hamiltonian for one heavy-light meson:

$$
H^{(1)}=\frac{p^{2}}{2 m}-\frac{2\left(N_{c}-1\right)\left(N_{c}+1\right)}{N_{c}} V(\vec{r})
$$

where $p$ and $\vec{r}$ are the light quark momentum and position. This means that to leading order in $N_{c}$ the Hamiltonian (31) corresponds just to the sum of two non interacting mesons superposed at the origin. This corresponds to the factorization assumption.

Eq. (31) exhibits a symmetry for the exchange of spatial variables $\vec{r}_{\bar{\alpha}} \rightarrow \vec{r}_{\bar{\beta}}$. This symmetry is simply a product of the color permutation symmetry $P_{c}$, valid at $t=0$, and the global permutation symmetry $P$, valid for all $t$. The eigenstates of (31) are eigenstates of the spatial permutation $\vec{r}_{\bar{\alpha}} \rightarrow \vec{r}_{\bar{\beta}}$, and it is not difficult to guess that the ground states are symmetric under the latter permutation. This is illustrated in the Appendix.

Hence, restricting ourselves to the subspace spanned by the two fundamental states, which are symmetric states for the permutation $\vec{r}_{\bar{\alpha}} \rightarrow \vec{r}_{\bar{\beta}}$, we have $P_{c}=P$ and we project the state $\mid f>$, (5), into the subspace

$$
\mathcal{H}_{0}=\left|D^{+, 0,0}, 0>\oplus\right| D^{-, 0,0}, 0>
$$

We have:

$$
<D^{ \pm, 0,0} 0 \mid f>=\frac{\left(C_{1} \pm C_{2}\right)\left(1 \pm \frac{1}{N_{c}}\right)}{\left(2 \pm \frac{2}{N_{c}}\right)^{\frac{1}{2}}} S^{ \pm}
$$

where $S^{ \pm}$is the spatial overlap. As stated above $S^{ \pm}=S^{0}+O\left(1 / N_{c}\right)$ where $S^{0}$ is the spatial overlap of $\mid f>$ with the direct product of two non interacting mesons located at the origin, i.e. $\psi^{(1)}\left(\vec{r}_{\bar{\alpha}}\right) \psi^{(1)}\left(\vec{r}_{\bar{\beta}}\right), \psi^{(1)}(\vec{r})$ being the ground-state eigenfunction of $H^{(1)}(32)$.

The evolution forward in time of the states $\mid D^{ \pm, 0,0}, t>$ is obtained by replacing in (26) the $-\infty$ lower bounds of the integrals by 0 . It results, thanks to time reversal, in phase shifts which are simply divided by $2: e^{i \delta \pm i \phi}$. 
The resulting $T$ matrix for the decay of the initial meson $P_{A \bar{\alpha}}$ into the fundamental mesons is:

$$
\begin{aligned}
& T\left(P_{A \bar{\alpha}} \rightarrow M_{C \bar{\beta}}^{(0)} M_{B \bar{\alpha}}^{(0)}\right)=e^{i \delta}\left\{\frac{\left(C_{1}+C_{2}\right)\left(1+\frac{1}{N_{c}}\right) S^{+}}{2\left(1+\frac{1}{N_{c}}\right)^{\frac{1}{2}}} e^{i \phi}+\frac{\left(C_{1}-C_{2}\right)\left(1-\frac{1}{N_{c}}\right) S^{-}}{2\left(1-\frac{1}{N_{c}}\right)^{\frac{1}{2}}} e^{-i \phi}\right\} \\
& T\left(P_{A \bar{\alpha}} \rightarrow M_{C \bar{\alpha}}^{(0)} M_{B \bar{\beta}}^{(0)}\right)=e^{i \delta}\left\{\frac{\left(C_{1}+C_{2}\right)\left(1+\frac{1}{N_{c}}\right) S^{+}}{2\left(1+\frac{1}{N_{c}}\right)^{\frac{1}{2}}} e^{i \phi}-\frac{\left(C_{1}-C_{2}\right)\left(1-\frac{1}{N_{c}}\right) S^{-}}{2\left(1-\frac{1}{N_{c}}\right)^{\frac{1}{2}}} e^{-i \phi}\right\}
\end{aligned}
$$

To perform a systematic $1 / N_{c}$ expansion, let us first define $\Delta S^{ \pm}$by

$$
S^{ \pm} \equiv S^{0}+\frac{\Delta S^{ \pm}}{N_{c}}
$$

Then, from (35) we obtain to first order in $1 / N_{c}$ :

$$
\begin{aligned}
T\left(P_{A \bar{\alpha}} \rightarrow M_{C \bar{\beta}}^{(0)} M_{B \bar{\alpha}}^{(0)}\right)= & e^{i \delta} S^{0}\left\{\left(C_{1}+\frac{C_{2}}{2 N_{c}}+\frac{C_{1}\left(\Delta S^{+}+\Delta S^{-}\right)}{2 N_{c} S^{0}}\right) \cos \phi\right. \\
& \left.+i\left(C_{2}+\frac{C_{1}}{2 N_{c}}+\frac{C_{2}\left(\Delta S^{+}+\Delta S^{-}\right)}{2 N_{c} S^{0}}\right) \sin \phi\right\} \\
T\left(P_{A \bar{\alpha}} \rightarrow M_{C \bar{\alpha}}^{(0)} M_{B \bar{\beta}}^{(0)}\right)= & e^{i \delta} S^{0}\left\{\left(C_{2}+\frac{C_{1}}{2 N_{c}}+\frac{C_{2}\left(\Delta S^{+}+\Delta S^{-}\right)}{2 N_{c} S^{0}}\right) \cos \phi\right. \\
& \left.+i\left(C_{1}+\frac{C_{2}}{2 N_{c}}+\frac{C_{1}\left(\Delta S^{+}+\Delta S^{-}\right)}{2 N_{c} S^{0}}\right) \sin \phi\right\}
\end{aligned}
$$

Comparing (37) with eqs (12) and (14) we see that if we take in (37) $\Delta S^{ \pm}=$ $\phi=0$ we recover the factorization corrected à la Shifman, i.e. with eq. (16). The global phase $\delta$ is not relevant here. Consequently we learn that this factor $1 / 2$ à la Shifman is completed by two other effects at the same order in $1 / N_{c}$ : i) the $\Delta S^{ \pm}$ which reflect the difference, at the time of the weak decay, between the total $q q \bar{q} \bar{q}$ spatial wave function and the simple product of the two asymptotic meson wave functions; ii) the phase $\phi$ which reflects strictly speaking the final state interaction. The latter phase $\phi$, although $1 / N_{c}$ suppressed may become very large when $v \rightarrow 0$. For $\phi=\pi / 4$ the factorization becomes grossly wrong since the role of the two final states is interchanged: The operator multiplying $C_{1}$ in $H_{W}$ produces dominantly the $M_{C \bar{\alpha}}^{(0)} M_{B \bar{\beta}}^{(0)}$ instead of $M_{C \bar{\beta}}^{(0)} M_{B \bar{\alpha}}^{(0)}$ as suggested by factorization, and vice versa for $C_{2}$. In other words, the class II decays become dominant over the class I when $\phi=\pi / 4$.

Furthermore, the dynamical origin of the phase $\phi$ and of the factors $\Delta S^{ \pm}$is obviously strongly dependent of the precise nature of the decay channels considered. This indicates that the effects of $\phi$ and $\Delta S^{ \pm}$cannot be incorporated in a phenomenological factorization à la BSW, which assumes a given pair of constants $a_{1}$ and $a_{2}$ for all the decay channels of a meson.

In the appendix we have performed an explicit calculation of $\Delta S^{ \pm}$and an estimation of $\phi$ for an harmonic oscillator potential. As can be seen from (71) and 74), $\phi$ is always the dominant $1 / N_{c}$ correction when $\bar{\alpha}$ and $\bar{\beta}$ are light quarks, and when they are heavy, $\phi$ still dominates as long as $v m R \leq 1$.

Let us now consider a more general case by first introducing spin. 


\subsection{Final state interactions of the fundamental pseudoscalar and vector mesons.}

From heavy quark symmetry (HQS) we know that vector and pseudoscalar mesons are degenerate. HQS also tells us that the heavy quark spin is conserved. This is of course a trivial consequence of (20), but it is quite general. For example, in (20) we might add a term coupling the light quark spins, such as $\vec{\sigma}_{\bar{\beta}} \cdot \vec{\sigma}_{\bar{\alpha}}$, but all terms including the spins $s_{B}$ and $s_{C}$ are $1 / M$ suppressed.

Restricting ourselves to a 0 total quark spin, the ground states combine into four possible asymptotic states: $P_{C \bar{\beta}} P_{B \bar{\alpha}}, V_{C \bar{\beta}} V_{B \bar{\alpha}}, P_{B \bar{\beta}} P_{C \bar{\alpha}}$ and $V_{B \bar{\beta}} V_{C \bar{\alpha}}$ (where $P$ stands for pseudoscalar and $V$ for vector).

It is then convenient to use states with a given symmetry for $P_{S} \equiv s_{B} \leftrightarrow s_{C}$. The relevant combinations are:

$$
\begin{gathered}
P_{S}\left\{\frac{-\left|P_{C \bar{\beta}} P_{B \bar{\alpha}}>+\right| V_{C \bar{\beta}} V_{B \bar{\alpha}}>}{2}\right\}=-\left\{\frac{-\left|P_{C \bar{\beta}} P_{B \bar{\alpha}}>+\right| V_{C \bar{\beta}} V_{B \bar{\alpha}}>}{2}\right\} \\
P_{S}\left\{\frac{-3\left|P_{C \bar{\beta}} P_{B \bar{\alpha}}>-\right| V_{C \bar{\beta}} V_{B \bar{\alpha}}>}{2}\right\}=\left\{\frac{-3\left|P_{C \bar{\beta}} P_{B \bar{\alpha}}>-\right| V_{C \bar{\beta}} V_{B \bar{\alpha}}>}{2}\right\}
\end{gathered}
$$

where $V V^{\prime}$ stands for $V^{0} V^{\prime 0}-V^{+} V^{\prime-}-V^{-} V^{\prime+}$ with $0,+,-$ labelling the polarization of the vector mesons.

In fact, the first combination in (38) corresponds to $S_{B C}=0$ (total spin of $B$ and $C$ ) and the second to $S_{B C}=1$. Using for large $|T|$ the notation:

$$
\begin{aligned}
& \mid{ }^{1} D_{C \bar{\beta} ; B \bar{\alpha}}^{(0,0)}, T>=\left\{\frac{-\left|P_{C \bar{\beta}}(-\vec{v} T) P_{B \bar{\alpha}}(\vec{v} T)>+\right| V_{C \bar{\beta}}(-\vec{v} T) V_{B \bar{\alpha}}(\vec{v} T)>}{2}\right\} \\
& \mid{ }^{3} D_{C \bar{\beta} ; B \bar{\alpha}}^{(0,0)}, T>=\left\{\frac{-3\left|P_{C \bar{\beta}}(-\vec{v} T) P_{B \bar{\alpha}}(\vec{v} T)>-\right| V_{C \bar{\beta}}(-\vec{v} T) V_{B \bar{\alpha}}(\vec{v} T)>}{2}\right\}
\end{aligned}
$$

the strong Hamiltonian is diagonal in the basis where both $P_{S}$ and $P \equiv \bar{\alpha} \leftrightarrow \bar{\beta}$ are diagonal:

$$
\begin{array}{ll}
\left.\sqrt{2}\right|^{1} D^{ \pm, 0,0}, T>\underset{\substack{T \rightarrow \pm \infty \\
=}}{=}\left|{ }^{1} D_{C \bar{\beta} ; B \bar{\alpha}}^{(0,0)}, T> \pm\right|^{1} D_{C \bar{\alpha} ; B \bar{\beta}}^{(0,0)}, T> \\
\left.\sqrt{2}\right|^{3} D^{ \pm, 0,0}, T>\underset{\substack{=\\
T \rightarrow \pm \infty}}{=} & \left|{ }^{3} D_{C \bar{\beta} ; B \bar{\alpha}}^{(0,0)}, T> \pm\right|^{3} D_{C \bar{\alpha} ; B \bar{\beta}}^{(0,0)}, T>
\end{array}
$$

The four states in (40) evolve diagonally under the strong Hamiltonian and lead to four phase shifts given by formulae similar to eq. (26).

Next we make the assumption that $\mid f>$ defined in (5) is odd under $P_{S}$ :

$$
P_{S}|f>=-| f>
$$

Relation (41) is a consequence of Fierz symmetry whenever $H_{W}$ is build up of Fierz-invariant currents, as is the case in eq. (4). The fact that Fierz symmetry 
translates into a spin antisymmetry as in (41) comes from the fact that Fierz transformation contains an additional minus sign from fermion field commutation. It results that only the states $\mid{ }^{1} D^{ \pm, 0,0}, 0>$ are produced during weak decay, i.e. $S_{B C}=0$. The arguments from the beginning of section 3.2 to eq. (34) may be repeated, except that due to the spin asymetry, the $\mid{ }^{1} D^{+, 0,0}, 0>\left(\mid{ }^{1} D^{-, 0,0}, 0>\right.$ is color-odd (color-even):

$$
<D^{ \pm, 0,0} 0 \mid f>=\frac{\left(C_{1} \mp C_{2}\right)\left(1 \mp \frac{1}{N_{c}}\right)}{\left(2 \mp \frac{2}{N_{c}}\right)^{\frac{1}{2}}} S^{ \pm}
$$

leading to

$$
\begin{aligned}
& T\left(P_{A \bar{\alpha}} \rightarrow^{1} D_{C \bar{\beta} ; B \bar{\alpha}}^{(0,0)}\right)=e^{i \delta}\left\{\frac{\left(C_{1}-C_{2}\right)\left(1-\frac{1}{N_{c}}\right) S^{+}}{2\left(1-\frac{1}{N_{c}}\right)^{\frac{1}{2}}} e^{i \phi}+\frac{\left(C_{1}+C_{2}\right)\left(1+\frac{1}{N_{c}}\right) S^{-}}{2\left(1+\frac{1}{N_{c}}\right)^{\frac{1}{2}}} e^{-i \phi}\right\} \\
& T\left(P_{A \bar{\alpha}} \rightarrow^{1} D_{C \bar{\alpha} ; B \bar{\beta}}^{(0,0)}=e^{i \delta}\left\{\frac{\left(C_{1}-C_{2}\right)\left(1-\frac{1}{N_{c}}\right) S^{+}}{2\left(1-\frac{1}{N_{c}}\right)^{\frac{1}{2}}} e^{i \phi}-\frac{\left(C_{1}+C_{2}\right)\left(1+\frac{1}{N_{c}}\right) S^{-}}{2\left(1+\frac{1}{N_{c}}\right)} e^{\frac{1}{2}} e^{-i \phi}\right\}\right.
\end{aligned}
$$

and to first order in $1 / N_{c}$ :

$$
\begin{gathered}
T\left(P_{A \bar{\alpha}} \rightarrow M_{C \bar{\beta}}^{(0)} M_{B \bar{\alpha}}^{(0)}\right)=\quad \frac{\eta}{2} e^{i \delta} S^{0}\left\{\left(C_{1}+\frac{C_{2}}{2 N_{c}}+\frac{C_{1}\left(\Delta S^{+}+\Delta S^{-}\right)}{2 N_{c} S^{0}}\right) \cos \phi\right. \\
\left.-i\left(C_{2}+\frac{C_{1}}{2 N_{c}}+\frac{C_{2}\left(\Delta S^{+}+\Delta S^{-}\right)}{2 N_{c} S^{0}}\right) \sin \phi\right\} \\
T\left(P_{A \bar{\alpha}} \rightarrow M_{C \bar{\alpha}}^{(0)} M_{B \bar{\beta}}^{(0)}\right)=\frac{\eta}{2} e^{i \delta} S^{0}\left\{-\left(C_{2}+\frac{C_{1}}{2 N_{c}}+\frac{C_{2}\left(\Delta S^{+}+\Delta S^{-}\right)}{2 N_{c} S^{0}}\right) \cos \phi\right. \\
\left.+i\left(C_{1}+\frac{C_{2}}{2 N_{c}}+\frac{C_{1}\left(\Delta S^{+}+\Delta S^{-}\right)}{2 N_{c} S^{0}}\right) \sin \phi\right\}
\end{gathered}
$$

where $\eta=+1$ for longitudinal vector mesons and $\eta=-1$ for transverse vector and pseudoscalar mesons. The difference between the r.h.s of (43) and the r.h.s. of (35) comes from the interchange of color symmetric with color antisymmetric combinations as apparent when comparing (34) and (42).

It is to be noted that the relation between $P P$ and $V V$ production amplitude is exactly given by the fact that only the ${ }^{1} D$ combination are created. Indeed, this relation is a consequence of HQS and would only be corrected if we considered the $O(1 / M)$ corrections. This relation is not a surprise when one realizes that the conditions (11) and (2) imply an S-wave dominated decay.

\subsection{Semi-inclusive decay.}

We have found important channel dependent corrections to factorization. We may still wonder if these corrections ares not washed out when we sum up on one side all the decay channels $M_{C \bar{\beta}}^{(n)} M_{B \bar{\alpha}}^{(m)}$ for all $m, n$, and on the other side all the channels $M_{B \bar{\beta}}^{(n)} M_{C \bar{\alpha}}^{(m)}$. This is the aim of this section.

Let us call $\mathcal{H}^{+}(t)\left(\mathcal{H}^{-}(t)\right)$ the Hilbert space spanned by the set of states $\mid D^{+, n, m}, t>, \forall n, m\left(\mid D^{-, n, m}, t>, \forall n, m\right)$ defined in eq. (21). $\mathcal{H}^{+}(t)\left(\mathcal{H}^{-}(t)\right)$ contains 
the even (odd) states under the permutation $P \equiv \bar{\alpha} \leftrightarrow \bar{\beta}$. The latter commuting with the Hamiltonian $H(t)$, the evolution does not mix the spaces $\mathcal{H}^{+}(t)$ and $\mathcal{H}^{-}(t)$. We shall call $U^{+}\left(t_{1}, t_{2}\right)\left(U^{-}\left(t_{1}, t_{2}\right)\right)$ the evolution operator in $\mathcal{H}^{+}(t)\left(\mathcal{H}^{-}(t)\right)$. $U^{ \pm}\left(t_{1}, t_{2}\right)$ are unitary.

As already stated in section 3.2, $H(0)$ is also invariant under the color permutation $P_{c}: C_{C} \leftrightarrow C_{B}$. For $t=0$ the Permutation $P=P_{c} P_{r}$ where $P_{r} \equiv p_{\bar{\alpha}}, s_{\bar{\alpha}} \leftrightarrow p_{\bar{\beta}}, s_{\bar{\beta}}$ (remember $\vec{r}_{B}=\vec{r}_{C}=0$ for $t=0$ ). We then decompose $\mid f>$ both into eigenstates of $P$ :

$$
\begin{aligned}
& \left|f^{+}>=\frac{\left(C_{1}+C_{2}\right)\left(\delta_{c_{C}, c_{\bar{\beta}}} \delta_{c_{B}, c_{\bar{\alpha}}}+\delta_{c_{C}, c_{\bar{\alpha}}} \delta_{c_{B}, c_{\bar{\beta}}}\right)}{2 N_{c}}\right| f_{+}^{S}>+\frac{\left(C_{1}-C_{2}\right)\left(\delta_{c_{C}, c_{\bar{\beta}}} \delta_{c_{B}, c_{\bar{\alpha}}}-\delta_{c_{C}, c_{\bar{\alpha}}} \delta_{c_{B}, c_{\bar{\beta}}}\right)}{2 N_{c}} \mid f_{-}^{S}>
\end{aligned}
$$

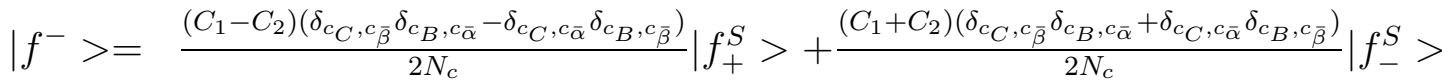

where $\mid f^{ \pm}>$are eigenstates of $P$ with eigenvalue,$\pm \mid f_{S}>$ contains the spin-space part of the wave function (5):

$$
\begin{gathered}
\mid f^{S}>=\sum_{s_{C}, s_{\bar{\beta}}, c_{C}, c_{\bar{\beta}},} \int d \vec{p}_{C} d \vec{p}_{\bar{\beta}} d \vec{p}_{B} d \vec{p}_{\bar{\alpha}} \Psi\left(\vec{p}_{C}, s_{C}, \vec{p}_{\bar{\beta}}, s_{\bar{\beta}}, \vec{p}_{B}, s_{B}, \vec{p}_{\bar{\alpha}}, s_{\bar{\alpha}}\right) \\
s_{B}, s_{\bar{\alpha}}, c_{B}, c_{\bar{\alpha}} \\
\mid C, \vec{p}_{C}, s_{C}, c_{C} ; \bar{\beta}, \vec{p}_{\bar{\beta}}, s_{\bar{\beta}}, c_{\bar{\beta}} ; B, \vec{p}_{B}, s_{B}, c_{B} ; \bar{\alpha}, \vec{p}_{\bar{\alpha}}, s_{\bar{\alpha}}, c_{\bar{\alpha}}>
\end{gathered}
$$

which is expanded into the eigenvectors of $P_{r}, \mid f_{ \pm}^{S}>$ corresponding to eigenvalues $P_{r}\left|f_{ \pm}^{S}>= \pm\right| f_{ \pm}^{S}>$.

The norm of $\mid f^{ \pm}>$is:

$$
<f^{ \pm} \mid f^{ \pm}>=\frac{1}{2}\left[\left(C_{1} \pm C_{2}\right)^{2}\left(1 \pm \frac{1}{N_{c}}\right)<f_{+}^{S}\left|f_{+}^{S}>+\left(C_{1} \mp C_{2}\right)^{2}\left(1 \mp \frac{1}{N_{c}}\right)<f_{-}^{S}\right| f_{-}^{S}>\right]
$$

If we define $<f_{ \pm}^{S} \mid f_{ \pm}^{S}>\equiv K_{ \pm}$, then $K_{+}+K_{-}=K$ as defined in eq. (7).

As stated above:

$$
\mid f^{ \pm}>\in \mathcal{H}^{ \pm}
$$

The evolution toward a large positive time $T$ leads to:

$$
\left|f^{ \pm}(T)>=U^{ \pm}(T, 0)\right| f^{ \pm}>
$$

By unitarity the norm of $\mid f^{ \pm}(T)>$ equals that of $\mid f^{ \pm}>$.

We get

$$
\begin{aligned}
\sqrt{2} T\left(P_{A \bar{\alpha}} \rightarrow D^{ \pm, m, n}\right)= & \left(C_{1} \pm C_{2}\right)\left(1 \pm \frac{1}{N_{c}}\right)^{\frac{1}{2}} K_{+}^{\frac{1}{2}} S_{+}^{ \pm, m, n} e^{i \phi_{+}^{ \pm, m, n}}+ \\
& \left(C_{1} \mp C_{2}\right)\left(1 \mp \frac{1}{N_{c}}\right)^{\frac{1}{2}} K_{-}^{\frac{1}{2}} S_{-}^{ \pm, m, n} e^{i \phi_{-}^{ \pm, m, n}}
\end{aligned}
$$


where $S_{ \pm}^{ \pm, m, n}$ and $\phi_{ \pm}^{ \pm, m, n}$ are real numbers defined by:

$$
S_{ \pm}^{ \pm, m, n} e^{i \phi_{ \pm}^{ \pm, m, n}}=\frac{1}{\left(<f^{ \pm}(T) \mid f^{ \pm}(T)>\right)^{\frac{1}{2}}}<D^{ \pm, m, n}, T \mid f_{ \pm}^{ \pm}(T)>
$$

with

$$
f_{ \pm}^{ \pm}(T)=U^{ \pm}(T, 0) \mid f_{ \pm}^{ \pm}(0)>
$$

$f_{+}^{ \pm}\left(f_{-}^{ \pm}\right)$being the first (second) terms in the right hand sides of (45).

From unitarity:

$$
\sum_{m, n}\left(S_{+}^{ \pm, m, n}\right)^{2}+\left(S_{-}^{ \pm, m, n}\right)^{2}=1
$$

which leads to

$$
\sum_{ \pm, m, n}\left|T\left(P_{A \bar{\alpha}} \rightarrow D^{ \pm, n, m}\right)\right|^{2}=\left(C_{1}^{2}+C_{2}^{2}+2 \frac{C_{1} C_{2}}{N_{c}}\right)
$$

as expected from (6) and unitarity.

Finally

$$
\begin{aligned}
T\left(P_{A \bar{\alpha}} \rightarrow M_{C \bar{\beta}}^{(m)} M_{B \bar{\alpha}}^{(n)}\right)= & K_{+}^{\frac{1}{2}}\left\{\frac{\left(C_{1}+C_{2}\right)\left(1+\frac{1}{N_{c}}\right) S_{+}^{+, m, n}}{2\left(1+\frac{1}{N_{c}}\right)^{\frac{1}{2}}} e^{i \phi_{+}^{+, m, n}}+\frac{\left(C_{1}-C_{2}\right)\left(1-\frac{1}{N_{c}}\right) S_{+}^{-, m, n}}{2\left(1-\frac{1}{N_{c}}\right)^{\frac{1}{2}}} e^{i \phi_{+}^{-, m, n}}\right\} \\
& K_{-}^{\frac{1}{2}}\left\{\frac{\left(C_{1}-C_{2}\right)\left(1-\frac{1}{N_{c}}\right) S_{-}^{+, m, n}}{2\left(1-\frac{1}{N_{c}}\right){ }^{\frac{1}{2}}} e^{i \phi_{-}^{+, m, n}}+\frac{\left(C_{1}+C_{2}\right)\left(1+\frac{1}{N_{c}}\right) S_{-}^{-, m, n}}{2\left(1+\frac{1}{N_{c}}\right)^{\frac{1}{2}}} e^{i \phi_{-}^{-, m, n}}\right\} \\
T\left(P_{A \bar{\alpha}} \rightarrow M_{C \bar{\alpha}}^{(m)} M_{B \bar{\beta}}^{(n)}=\right. & K_{+}^{\frac{1}{2}}\left\{\frac{\left(C_{1}+C_{2}\right)\left(1+\frac{1}{N_{c}}\right) S_{+}^{+, m, n}}{2\left(1+\frac{1}{N_{c}}\right)} e^{\frac{1}{2}} \phi_{+}^{+, m, n}-\frac{\left(C_{1}-C_{2}\right)\left(1-\frac{1}{N_{c}}\right) S_{+}^{-, m, n}}{2\left(1-\frac{1}{N_{c}}\right)^{\frac{1}{2}}} e^{i \phi_{+}^{-, m, n}}\right\} \\
& K_{-}^{\frac{1}{2}}\left\{\frac{\left(C_{1}-C_{2}\right)\left(1-\frac{1}{N_{c}}\right) S_{-}^{+, m, n}}{2\left(1-\frac{1}{N_{c}}\right)} e^{\frac{1}{2}}{\phi_{-}^{+, m, n}}^{\frac{1}{2}}-\frac{\left(C_{1}+C_{2}\right)\left(1+\frac{1}{N_{c}}\right) S_{-}^{-, m, n}}{2\left(1+\frac{1}{N_{c}}\right)^{\frac{1}{2}}} e^{i \phi_{-}^{-, m, n}}\right\}
\end{aligned}
$$

Duality is fully verified since summing over all states

$$
\sum_{m, n}\left|T\left(P_{A \bar{\alpha}} \rightarrow M_{C \bar{\beta}}^{(m)} M_{B \bar{\alpha}}^{(n)}\right)\right|^{2}+\left|T\left(P_{A \bar{\alpha}} \rightarrow M_{C \bar{\alpha}}^{(m)} M_{B \bar{\beta}}^{(n)}\right)\right|^{2}=\left(C_{1}^{2}+C_{2}^{2}+2 \frac{C_{1} C_{2}}{N_{c}}\right) K .
$$

let us now consider the partially inclusive sums:

$$
\begin{aligned}
& \sum_{m, n}\left|T\left(P_{A \bar{\alpha}} \rightarrow M_{C \bar{\beta}}^{(m)} M_{B \bar{\alpha}}^{(n)}\right)\right|^{2} \equiv \Sigma_{C \bar{\beta} ; B \bar{\alpha}} \\
& \sum_{m, n}\left|T\left(P_{A \bar{\alpha}} \rightarrow M_{C \bar{\alpha}}^{(m)} M_{B \bar{\beta}}^{(n)}\right)\right|^{2} \equiv \Sigma_{C \bar{\alpha} ; B \bar{\beta}}
\end{aligned}
$$


Nothing general can be said. In the $N_{c} \rightarrow \infty$ limit,

$$
S_{ \pm}^{+, m, n} \simeq S_{ \pm}^{-, m, n} ; \quad \phi_{ \pm}^{+, m, n} \simeq \phi_{ \pm}^{-, m, n}
$$

Assuming that for finite $N_{c}$ we keep the relations (58), we recover Shifman's Ansatz:

$$
\begin{aligned}
& \Sigma_{C \bar{\beta} ; B \bar{\alpha}}=\frac{K}{4}\left\{\left(C_{1}-C_{2}\right)\left(1-\frac{1}{N_{c}}\right)^{\frac{1}{2}}+\left(C_{1}+C_{2}\right)\left(1+\frac{1}{N_{c}}\right)^{\frac{1}{2}}\right\}^{2}=K\left\{C_{1}^{2}+\frac{C_{1} C_{2}}{N_{c}}+O\left(\frac{1}{N_{c}^{2}}\right)\right\} \\
& \Sigma_{C \bar{\alpha} ; B \bar{\beta}}=\frac{K}{4}\left\{\left(C_{1}-C_{2}\right)\left(1-\frac{1}{N_{c}}\right)^{\frac{1}{2}}-\left(C_{1}+C_{2}\right)\left(1+\frac{1}{N_{c}}\right)^{\frac{1}{2}}\right\}^{2}=K\left\{C_{2}^{2}+\frac{C_{1} C_{2}}{N_{c}}+O\left(\frac{1}{N_{c}^{2}}\right)\right\}
\end{aligned}
$$

If we made the opposite assumption that the relative phases $\phi_{ \pm}^{+, m, n}-\phi_{ \pm}^{-, m, n}$ are random, which might be reasonable at small velocity, the result would be

$$
\Sigma_{C \bar{\beta} ; B \bar{\alpha}}=\Sigma_{C \bar{\alpha} ; B \bar{\beta}}=K\left\{\frac{C_{1}^{2}+C_{2}^{2}}{2}+\frac{C_{1} C_{2}}{N_{c}}\right\}
$$

that is, an equal sharing of the total probability between the two sets of channels. In the latter case, even though we consider an inclusive sum, the final state interaction has a non trivial effect: the quarks have been redistributed at random between the final mesons. Of course such a random phase equal sharing may only happen when phase space allows for many final states to add up in a random way.

As a side remark we would like to mention another, not yet published, study that we have performed on duality versus factorization. We have considered a model with non-relativistic scalar quarks bound to color singlets by a color harmonic oscillator potential [10] without assuming heavy quarks neither using an adiabatic approximation as done in the present paper. This model also automatically restores duality i.e. the conservation of probability. Summing over all mesons in the limit in which the radius $R \rightarrow \infty$ one should find the free quark result. This is indeed the case:

$$
\begin{aligned}
& \Sigma_{C \bar{\beta} ; B \bar{\alpha}} \propto\left[C_{1}+y \frac{C_{2}}{N_{c}}\right]^{2}+O\left(\frac{1}{N_{c}^{2}}\right) \\
& \Sigma_{C \bar{\alpha} ; B \bar{\beta}} \propto\left[C_{2}+(1-y) \frac{C_{1}}{N_{c}}\right]^{2}+O\left(\frac{1}{N_{c}^{2}}\right)
\end{aligned}
$$

with $y$ depending non-trivially on the masses, unlike the universal factor $1 / 2$ proposed by Shifman. It is seen that FSI restores automatically duality. Notice by the way that expression (62), for an arbitrary value of $y$, is more general than Shifman's Ansatz and would as well restore duality.

\section{Conclusions.}

We have used a Quark Model where the motion of heavy quarks is treated as classical and where we assume two mass-degenerate antiquarks. We have used the resulting 
permutation symmetry to simplify the problem. We have restricted ourselves to the $q q \bar{q} \bar{q}$ sector, and we have shown that the contradiction between standard factorization and duality stems from the non-orthogonality in color space of the two decomposition of the $q q \bar{q} \bar{q}$ singlet into two pairs of $q \bar{q}$ color singlets. Taking care to use an orthogonal basis that diagonalizes the Hamiltonian, the dynamics of this sector shows very clearly how the final state interaction corrects standard factorization such as to satisfy duality.

Shifman [8] has proposed to correct factorization by a replacement of $1 / N_{c}$ by $1 /\left(2 N_{c}\right)$ while keeping the phenomenological factorization à la BSW. We have shown that this effect is indeed present. However we find two additional effects to same order in $1 / N_{c}$. One is related to the spatial distorsion of the meson wave functions at the time of the weak decay: the two mesons overlap in space and hence interact strongly. This has been expressed by our parameter $\Delta S^{ \pm}$. The second and more important additional effect is the phase difference $\phi$ between the permutationeven and permutation-odd states when evolving after the decay until the mesons are spatially distant. The latter effect is $O\left(1 / N_{c}\right)$ but also $O(1 / v)$ where $v$ is the final meson velocity in the total rest frame, and also $O(1 / m R)$ where $m$ is the light antiquarks constituant mass and $R$ is the wave function radius. This phase shift effect should dominate in the small velocity regime for light antiquarks. For large velocity and for heavy antiquarks, it vanishes.

We have seen that in the exclusive case, restricting ourselves to the ground state mesons, the phase shift $\phi$ is the dominant $1 / N_{c}$ correction for small velocity and light antiquarks, and it may produce a total modification of the factorization assumption, which could, for $\phi=\pi / 4$, be large enough to totally interchange the amplitudes of the two channels, and lead to a dominant class II decay. In an illustrative example treated in the appendix the $t=0$ wave function distortion , $\Delta S^{ \pm}$turns out to be small. We ignore if this is a general feature. Would it be so, it would indicate a validity of Shifman's Ansatz for large velocity and rather heavy antiquarks.

In the semi-inclusive case we compare the total decay probability into two sets of channels that correspond to the two possible pairing of $q q \bar{q} \bar{q}$ into two $q \bar{q}$. Again, the small phase shift, small distortion limit amounts to Shifman's Ansatz, while the opposite, random phase shift limit, amounts to an equal sharing of inclusive decay probability into the two sets of final channels. Again the latter situation may be reasonable in the small velocity case provided many final states are kinematically allowed.

\section{Acknowledgements.}

This work was supported in part by the CEC Science Project SC1-CT91-0729 and by the Human Capital and Mobility Programme, contract CHRX-CT93-0132. 


\section{A An illustrative example.}

As an illustration of the section 3.2, let us take for the potential $V$ in (20) an harmonic oscilator potential:

$$
V(\vec{r})=-\frac{N_{c}}{4\left(N_{c}^{2}-1\right) m R^{4}} \vec{r}^{2}
$$

so that the ground state solution of $(\sqrt{32})$ is:

$$
\psi^{(1)}(\vec{r})=\frac{1}{R^{\frac{3}{2}} \pi^{\frac{3}{4}}} e^{-\frac{r^{2}}{2 R^{2}}}
$$

where $r=|\vec{r}|$.

For the spatial part of the wave function $\mid f>$ we take in configuration space:

$$
\Psi\left(\vec{r}_{C}, \vec{r}_{\bar{\beta}}, \vec{r}_{B}, \vec{r}_{\bar{\alpha}}\right)=G \psi^{(1)}\left(\vec{r}_{\bar{\alpha}}\right) \delta_{3}\left(\vec{r}_{C}\right) \delta_{3}\left(\vec{r}_{B}\right) \delta_{3}\left(\vec{r}_{\bar{\beta}}\right)
$$

which expresses the fact that the weak operator is locall and that the quark $\bar{\alpha}$ is a spectator coming from the $P_{A \bar{\alpha}}$ meson, i.e. in the ground state wave function. $G$ is proportional to the Fermi constant.

To compute the spatial overlaps $S^{ \pm}$in (34), we need to know the ground state solutions of the Hamiltonian (31) with (63) for $V$. Let us change variables in (31):

$$
\begin{array}{rlrl}
\vec{r}_{\bar{\beta}} & =\vec{R}-\frac{1}{2} \vec{r}, & \vec{r}_{\bar{\alpha}}=\vec{R}+\frac{1}{2} \vec{r}, \\
\vec{p}_{\bar{\beta}}=\frac{1}{2} \vec{P}-\vec{p}, & p_{\bar{\alpha}}=\frac{1}{2} \vec{P}+\vec{p},
\end{array}
$$

leading to

$$
H(0)=\frac{P^{2}}{m}+\frac{p^{2}}{4 m}+\frac{\left(N_{c} \pm 2\right)\left(N_{c} \mp 1\right)}{4\left(N_{c}^{2}-1\right) m R^{4}} \vec{R}^{2}+\frac{\left(N_{c} \pm 4\right)\left(N_{c} \mp 1\right)}{\left(N_{c}^{2}-1\right) m R^{4}} \vec{r}^{2} .
$$

The ground state solution, i.e. the spatial wave functions of $\mid D^{ \pm, 0,0}, 0>$ is:

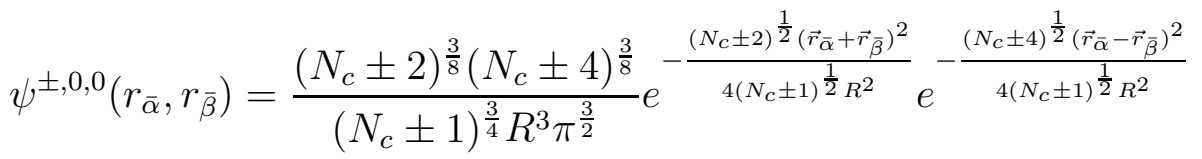

The overlap is given by

$$
\begin{aligned}
S^{ \pm} & =\int d^{3} r_{\bar{\alpha}} d^{3} r_{\bar{\beta}} G \psi^{(1) \dagger}\left(\vec{r}_{\bar{\alpha}}\right) \delta_{3}\left(\vec{r}_{\bar{\beta}}\right) \psi^{ \pm, 0,0}\left(r_{\bar{\alpha}}, r_{\bar{\beta}}\right) \\
& =G \frac{4^{\frac{3}{2}}\left(N_{c} \pm 2\right)^{\frac{3}{8}}\left(N_{c} \pm 4\right)^{\frac{3}{8}}}{\left[\left(N_{c} \pm 2\right)^{\frac{1}{2}}+\left(N_{c} \pm 4\right)^{\frac{1}{2}}+2\left(N_{c} \pm 1\right)^{\frac{1}{2}}\right]^{\frac{3}{2}} R^{\frac{3}{2}} \pi^{\frac{3}{4}}}
\end{aligned}
$$

\footnotetext{
${ }^{2}$ Remember that we assume here spinless quarks.
} 
where we have in (65) left aside the $\delta$ functions related to the heavy quarks, since the latter are treated classically. One has also for the factorization hypothesis result:

$$
S^{0}=G \frac{1}{R^{\frac{3}{2}} \pi^{\frac{3}{4}}}
$$

which is the $\psi^{(1)}(0)$ coming from the overlap of $\delta\left(\vec{r}_{\bar{\beta}}\right)$ with $\psi^{1}\left(\vec{r}_{\bar{\beta}}\right)$. The overlap of $\psi^{(1)}\left(\vec{r}_{\bar{\alpha}}\right)$ with itself gives obviously $1 . S^{0}$ in $(70)$ is obviously the $N_{c} \rightarrow \infty$ limite of $S^{ \pm}$in (69).

To next to leading order in $1 / N_{c}$ the calculation of $\Delta S^{ \pm}$defined in (36) is now straightforward from (69) and (70):

$$
\frac{\Delta S^{ \pm}}{N_{c}}= \pm \frac{3}{8 N_{c}}
$$

which turns out to be rather small, mainly because the normalization factor compensates for a large part the modification of the integral.

From (67) we also get the ground state energy:

$$
E^{ \pm}(0)=\frac{3}{2 m R^{2}}\left\{\left(\frac{N_{c} \pm 2}{N_{c} \pm 1}\right)^{\frac{1}{2}}+\left(\frac{N_{c} \pm 4}{N_{c} \pm 1}\right)^{\frac{1}{2}}\right\}
$$

leading to

$$
E^{+}(0)-E^{-}(0)=\frac{6}{m R^{2} N_{c}}+O\left(\frac{1}{N_{c}^{2}}\right)
$$

To compute $\phi$ we need, (26), to know $E^{+}(z)-E^{-}(z)$ for all $z \neq 0$. This is not so easy to compute. We will simply use $(73)$ to make an order of magnitude estimate. We will assume $E^{+}(z)-E^{-}(z)$ to be equal by $E^{+}(0)-E^{-}(0)$ as long as the hadrons overlap, i.e. for $|z| \leq c R$ where $c$ is some number of order 1 ? Then

$$
\phi \sim \frac{3 c}{m v R N_{c}}
$$

Although purely indicative, this result, besides confirming that $\phi \propto 1 / v N_{c}$ also learns us that $m R$ is the dimensionless number that gives the scale. When $\bar{\alpha}$ and $\bar{\beta}$ are light quark, it is known that $m R \sim 1$. Comparing (74) with (71) and to the $1 /\left(2 N_{c}\right)$ correction à la Shifman, we check that in the exclusive case, the phase shift $\phi$ is the dominant $1 / N_{c}$ contribution.

\footnotetext{
${ }^{3}$ The wave function radius for the wave function (64) is $\left\langle\vec{r}^{2}\right\rangle=3 / 2 R^{2}$. Hence one might think of taking $c \simeq 3 / 2$.
} 


\section{References}

[1] M.A. Shifman, A.I. Vainshtein and V.I. Zakharov, Zh. Ekxp. Teor. Fiz. $\underline{72}, 1275$ (1977) [Sov. Phys. JETP 또, 670 1977)].

[2] M. Wirbel, B. Stech and M. Bauer, Z. Phys. 29 637 (1985); $\underline{\text { C34 }}, 103$ (1987).

[3] C. E. Carlson and J. Milana, WM-94-110, Bulletin Board: hep-ph: 9409261.

[4] R. Aleksan et al., Beauty 94 workshop, April 1994, Mont Saint Michel, France (to be published in Nucl. Inst. and meth. A), LPTHE Orsay 94/53 Bulletin Board: hep-ph 9406334; M. Gourdin, A.N. Kamal and X.Y. Pham, PAR/LPTHE/94-19; R. Aleksan, A. Le Yaouanc, L. Oliver, O. Pène and J.-C. Raynal, DAPNIA/SPP 94-24, LPTHE-Orsay 94/15, hep-ph 9408215; R. Aleksan, A. Le Yaouanc, L. Oliver, O. Pène and J.-C. Raynal, First Arctic Workshop on Future Physics and Accelerators, Saariselka, Finland, Aug. 1994, LPTHE-Orsay 94/105, hepph 9412229 .

[5] A.N. Kamal and T.N. Pham Phys. Rev. D50 (1994) 6849; D50 (1994) R1832.

[6] A. N. Kamal et al. Alberta Thy-08-95 hep-ph: 9503390.

[7] J.F. Donoghue Phys. Rev. D33 (1986) 1516.

[8] M.A. Shifman, Nucl. Phys. B388 (1992) 346.

[9] R. Aleksan, A. Le Yaouanc, L. Oliver, O. Pene and J.-C. Raynal, Phys. Lett. B316, 567 (1993).

[10] M.B. Gavela et al. Phys. Lett. $\underline{79 B}$ (1978) 459; $\underline{82}$ (1979) 431; A. Le Yaouanc et al. Phys. Rev. D42 (1990) 3123. 